\title{
Strategies for operative management of abdominal wall hernia after solid organ transplant
}

\author{
Devinder Singh', Luther Holton ${ }^{2}$, Lauren Antognoli², Salman Choudhry ${ }^{2}$ \\ 'Division of Plastic Surgery, University of Miami, Miller School of Medicine, Miami, FL 33136, USA. \\ 2Division of Plastic Surgery, Anne Arundel Medical Center, Annapolis, MD 21401, USA.
}

Correspondence to: Dr. Devinder Singh, Division of Plastic Surgery, University of Miami, Miller School of Medicine, 1150 NW 14th St \#701, Miami, FL 33136, USA. E-mail: dsingh.md@gmail.com

How to cite this article: Singh D, Holton L, Antognoli L, Choudhry S. Strategies for operative management of abdominal wall hernia after solid organ transplant. Plast Aesthet Res 2020;7:39. http://dx.doi.org/10.20517/2347-9264.2019.76

Received: 13 Feb 2020 First Decision: 23 Apr 2020 Revised: 27 May 2020 Accepted: 22 Jun 2020 Published: 19 Jul 2020

Academic Editor: Sahil Kuldip. Kapur Copy Editor: Cai-Hong Wang Production Editor: Jing Yu

\begin{abstract}
About $5 \%-11 \%$ of all abdominal surgery results in incisional hernia. This rate can be even higher among highrisk populations such as transplant patients. Lifetime incidence of incisional hernia following liver transplant is as high as $43 \%$ in recent studies. The transplant population is at higher risk for incisional hernia precisely because of their immunosuppressive therapy. Thus, it is imperative to understand the risk factors for incisional hernia in this unique patient population. This article focuses on understanding preoperative, intraoperative, and postoperative risk factors for failure of hernia repair in the transplant population in addition to discussing risk stratification for incisional hernia in this population. Furthermore, we discuss the utility of panniculectomy in abdominal organ transplantation. Additionally, we discuss the value of mesh placement in abdominal wall closure. Finally, we review the concept of vascularized composite allograft as a method for achieving abdominal wall closure for patients who have failed more traditional repairs and who are left with inadequate tissue for successful repair.
\end{abstract}

Keywords: Abdominal organ transplant, incisional hernia, vascularized composite allograft, panniculectomy, human acellular dermal matrix, porcine acellular dermal matrix

\section{INTRODUCTION}

Approximately 5\%-11\% of all abdominal surgery incisions result in incisional hernia, but this rate can exceed $30 \%$ in complex wounds among high-risk patients such as those undergoing solid organ

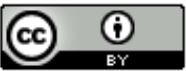

(C) The Author(s) 2020. Open Access This article is licensed under a Creative Commons Attribution 4.0 International License (https://creativecommons.org/licenses/by/4.0/), which permits unrestricted use, sharing, adaptation, distribution and reproduction in any medium or format, for any purpose, even commercially, as long as you give appropriate credit to the original author(s) and the source, provide a link to the Creative Commons license, and indicate if changes were made.

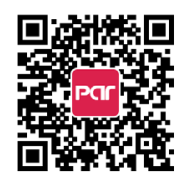


transplants ${ }^{[1]}$. The transplant population is uniquely susceptible to wound healing complications, such as wound infection and incisional hernia for multiple reasons. Lifetime incidence of incisional hernia following liver transplant has been reported to be as high as $43 \%$ in recent studies ${ }^{[2-4]}$. Wound complications can plague the postoperative patient experience as they cause discomfort, repeat hospitalizations, increased health care costs, and diminished quality of life. For transplant patients, the stakes are particularly high because wound infection, dehiscence, or incisional hernia have the potential to compromise graft function and viability and because efforts to heal are obtunded by the presence of often powerful immunosuppressive medications. Notably, efforts to modulate immunosuppression levels during events such as wound infections or malignancies have been thought to correlate with graft dysfunction and failure. Accordingly, preventing surgical site infection (SSI) is critical in these patients. Operative and perioperative strategies to prevent SSI and wound breakdown in the transplant population are similar to those of other surgical disciplines. In addition to maintaining a clean field with minimal spillage, use of drains, and a complex multi-layered closure of the fascia and subcutaneous tissue, for this particularly susceptible population, we have routinely used negative-pressure wound therapy in order to sequester the incision from outside contamination as well as aid perfusion across the incision. In fact, in May 2019, the Federal Drug Administration approved the PREVENA ${ }^{\mathrm{TM}}$ negative-pressure incision management system to help reduce superficial SSIs in patients at high risk of postoperative infections in Class I and II wounds. It is the first and only negative-pressure medical device indicated to aid in the reduction of SSIs. If a wound complication does occur, it should be addressed as early as possible to prevent progression. Active wound management with early debridement and washout can prevent worsening infection.

\section{ETIOLOGY AND RISK FACTORS}

\section{Immunosuppression}

Transplant patients are at especially high risk for hernia due to their immunosuppressed state and comorbid conditions which can hinder adequate healing and even hasten wound breakdown ${ }^{[1]}$. Induction immunosuppression is short-term, intense immunosuppression therapy administered to recipients at the time of transplantation in an attempt to prevent rejection in the first few weeks after transplant, when risk for rejection is highest. Modern induction immunosuppression in solid organ transplant recipients is not only focused on preventing rejection but also minimizing steroid use and mitigating the negative side effects of long-term immunosuppression. Current induction therapy commonly utilizes agents such as basiliximab, antithymocyte globulin, and alemtuzumab to reduce the use of steroids immediately after transplantation. The goal of maintenance therapy is to provide adequate long-term rejection prophylaxis through multiple immunosuppressive drugs with different mechanisms of action. Maintenance therapy often consists of tacrolimus, mycophenolate mofetil, and, at times, steroids. Vitamin A is also used as an adjunct to assist with wound healing in these patients on chronic steroids. Obviously, variation in induction and maintenance protocols exists between transplant centers, and all have potentially deleterious effects on wound healing. Aside from steroids used in induction and maintenance immunosuppression, steroid boluses are often used to treat patients experiencing rejection episodes. Thus, a lifetime of immunosuppression in transplant patients may allow their graft to survive, but at the cost of poor healing, wound breakdown, and hernias, which can potentially threaten the transplanted organ(s).

When planning elective surgery such as incisional hernia repair, careful consideration must be given to each patient's immunosuppressive regimen. A multidisciplinary approach is highly recommended for care for the transplant patient, including transplant pharmacologists to assist with drug modulation in the perioperative period. Decisions regarding adjustments to immunosuppressive therapy should be made on an individual and real-time basis and with the expertise of the involved team. Unfortunately, there are currently no data available from randomized, double-blind controlled clinical trials on how to guide immunosuppressive therapy in the perioperative setting for this patient population, making evidence-based 
recommendations difficult. Furthermore, chronic immunosuppression remains a cofactor for recurrence and subsequent hernia repairs, thus adding to the complexity of hernia repair in this population.

\section{Patient comorbidities}

Preoperative patient factors that predispose to incisional hernia in transplant patients include male gender, advanced age (studies cite ages greater than 45-60 as risk factors), elevated BMI (BMI > 25), smoking, malnutrition (serum albumin levels less than $3.5 \mathrm{~g} / \mathrm{L}$ ), connective tissue disorders (e.g., osteogenesis imperfecta, certain subtypes of Ehlers-Danlos syndrome, and Marfan syndrome), immediately preoperative chemotherapy and/or radiation to the operative site or region, presence of large volume ascites, COPD, previous surgery in the operative area, anemia, steroid use, diabetes mellitus, and immunosuppression ${ }^{[2-6]}$. Furthermore, patients with end-stage renal or liver disease live in catabolic states in which muscle atrophies and the abdominal wall weakens.

Postoperative factors associated with incisional hernia include wound infection, pulmonary complications, prolonged ICU stay, severe ascites, anemia, thrombocytopenia, acute rejection with steroid treatment, and same-site repeat surgery with fascial reopening and reclosure ${ }^{[2-6]}$.

Common to these risk factors - obesity, ascites, and COPD - is increased intra-abdominal pressure, which puts mechanical stress on the fascial closure and weakens the abdominal wall, making patients more prone to wound necrosis, breakdown, and hernia ${ }^{[2,4]}$. Furthermore, medical issues such as benign prostatic hypertrophy chronic constipation, and chronic cough can also significantly increase intra-abdominal pressure and thus jeopardize the integrity of hernia repairs. Lower abdominal incisions are also more prone to hernia, as increased abdominal pressure is greater in the dependent abdomen and a pannus may put tension on healing fascia. A patient undergoing abdominal organ transplant should be optimized to the greatest extent possible in the preoperative period. This should include careful medical management of weight, pulmonary function, and ascites to decrease postoperative intra-abdominal pressure, thus decreasing the incidence of developing incisional hernia. Unfortunately, patients often spend months and years on a transplant list and are often not adequately optimized for surgery when an organ actually becomes available.

\section{Technique-related risk factors}

Technique-related factors that increase risk for an incisional hernia after transplant include excess tension upon fascial closure, emergency surgery, and type of incision ${ }^{[2-6]}$. A surgeon's choice of incision for solid organ transplant surgery depends on the type of organ transplanted, surgeon preference, and patientspecific factors such as previous surgeries and organ size. The larger incisions with liver transplants are, in part, why liver transplant patients have a much higher incidence of hernia compared to renal transplant patients. Incisions commonly used in liver transplants include bilateral subcostal, Mercedes, and upper midline incisions, among multiple other variants [Figure 1]. Bilateral subcostal and Mercedes incisions are the most commonly used, as they provide excellent exposure. However, these large incisions with hours of stout retraction can lead to greater areas of fascial weakening, thus increased risk of future hernia. The Mercedes incision carries an increased risk of incisional hernia when compared to bilateral subcostal incisions ${ }^{[2]}$. The triple point of the Mercedes incision is a common area of decreased perfusion and increased tension, which can lead to wound ischemia, necrosis, and dehiscence at the levels of the fascia and/or the skin. Few studies exist regarding optimal closures for liver transplant incisions to prevent hernia. In a retrospective study from 2010, Aydin et al. ${ }^{[7]}$ described a unique approach for closure of abdominal fascia in Mercedes incisions, designed to reinforce the triple point. In this technique, the transverse components of the incision are closed in two overlapping layers, and the vertical component is closed in one layer using a single suture that overlaps the medial corners of the transverse incisions before running cephalad to the xiphoid. Patients whose fascia was closed in this method had significantly lower rates of 

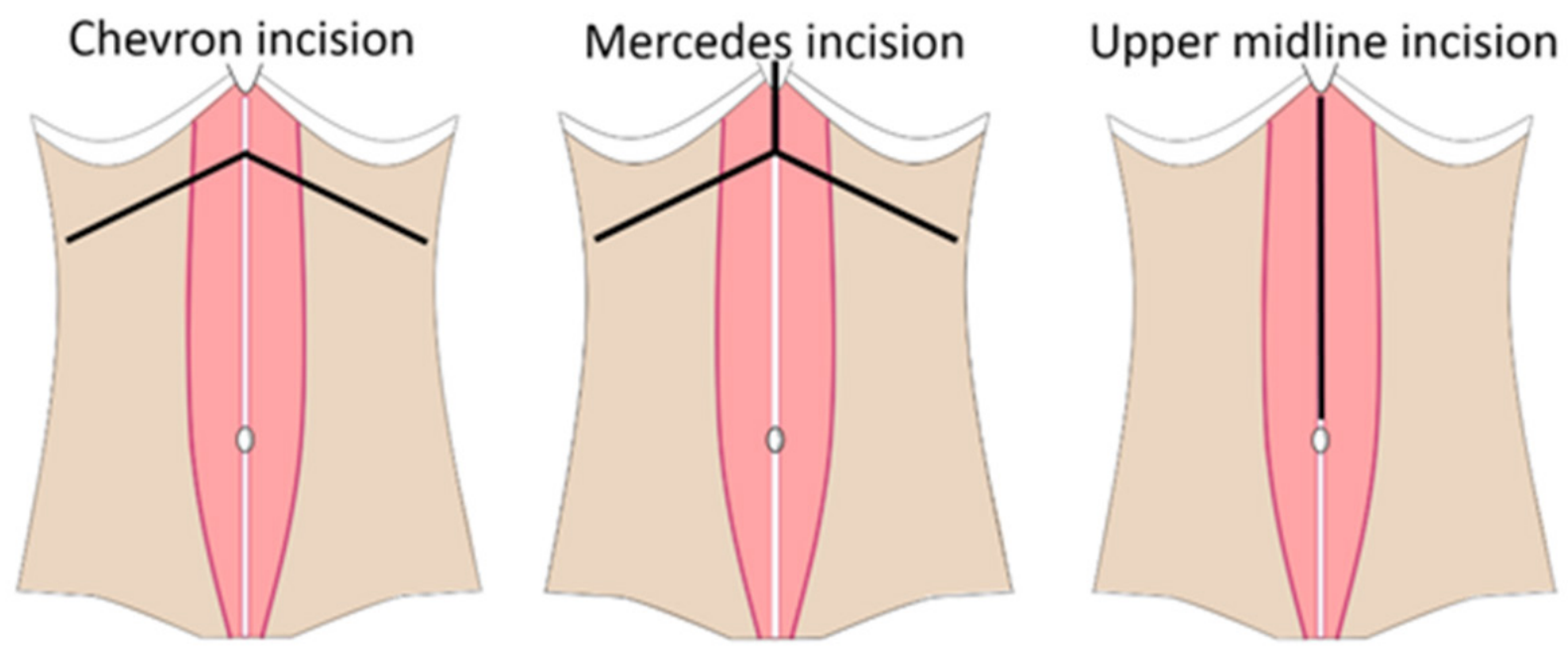

Figure 1. Examples of incisions used in liver transplant recipients

hernia than those closed in a single layer without this locking suture at the triple point. However, there is still no consensus on ideal closures for these large incisions, and the method of closure varies greatly between surgeons.

Common incisions described for kidney transplant recipients include paramedian, midline, and low oblique muscle-cutting incisions, such as the Rutherford - Morison incision [Figure 2]. Midline abdominal incisions are more prone to incisional hernia formation and carry a significantly higher risk of herniation compared to transverse and paramedian incisions ${ }^{[2]}$. Low oblique incisions result in fewer hernias than the "Hockey-stick" incision.

Finally, focused intraoperative assessment of fascia is essential. Although there may be concern for excessive fascial tension or even difficulty/inability to close the abdomen if there is not enough fascia, poor quality fascia must be debrided prior to closure. Including ischemic, nonviable fascia in a closure will greatly increase risk for wound breakdown, dehiscence, and exposure of the graft to infection.

\section{RISK STRATIFICATION}

Understanding how to avoid hernia complications in transplant patients is perhaps more valuable than understanding how to repair these hernias. Important measures to avoid hernia include active incision management (e.g., careful tissue handling, closing the operative area in multiple layers, placement of drains, and use of negative pressure incision management), as well as early identification of wound complications. Should wound complications occur, early management with operative debridement and washout as well as the use of adjunct modalities such as negative-pressure therapy with antibiotic solution irrigation may prove useful. However, hernias still occur in this complex patient population despite meticulous technique and early management of complications. Accordingly, risk stratification is helpful.

There is an established grading system created by the Ventral Hernia Working Group (VHWG) to predict surgical site occurances in patients undergoing ventral hernia repair based on patient risk factors and comorbidities. This was initially described in 2002 and consisted of four grades (low risk, comorbid, potentially contaminated, and infected). The grading system was then redefined in 2012, which resulted in Grades 3 and 4 being combined [Table 1] ${ }^{[8]}$. Transplant patients by virtue of immunosuppression are classified as at least Grade 2. 

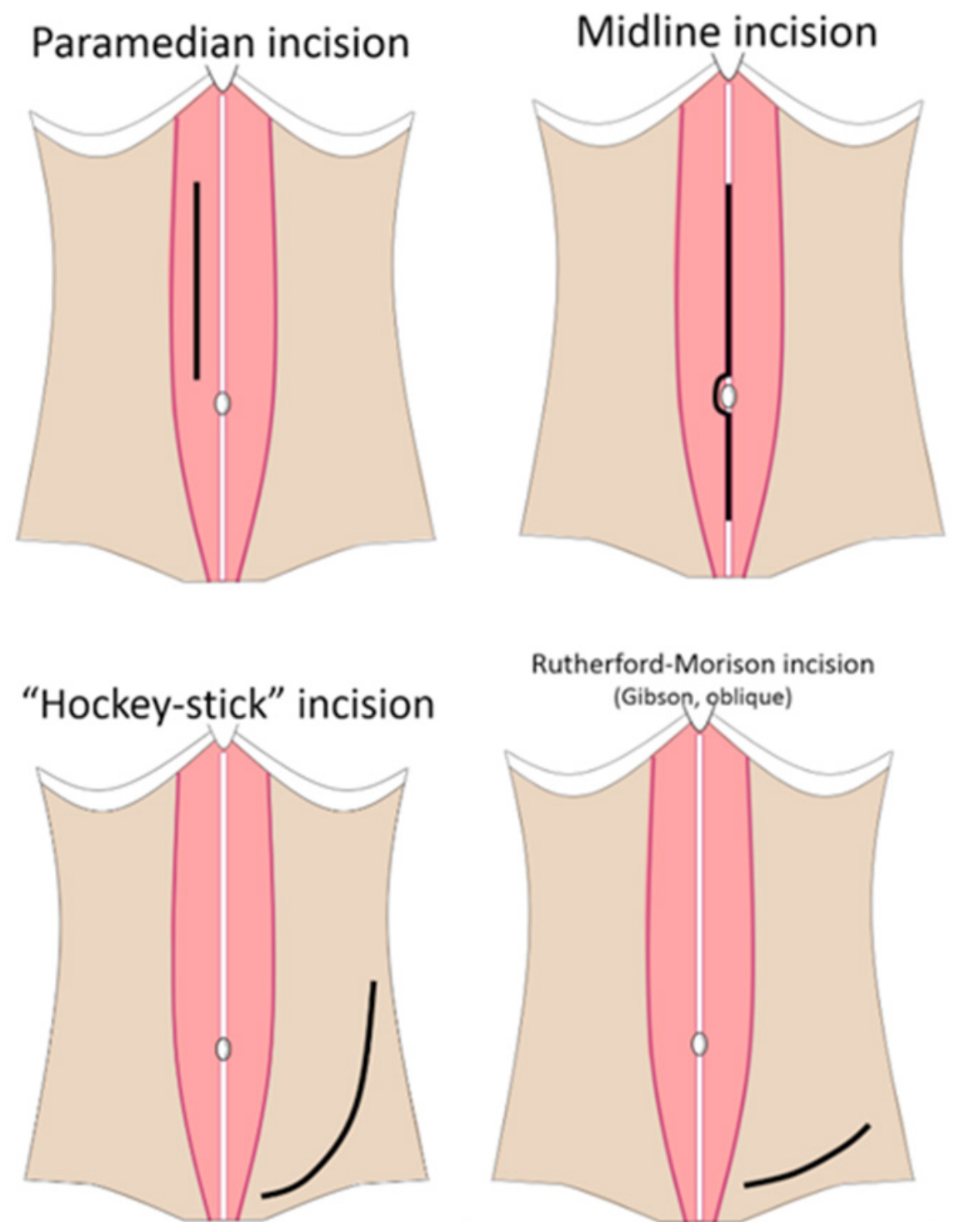

Figure 2. Examples of incisions used in kidney transplant recipients. Please note that some incisions may be right- or left-sided, depending on patient anatomy and involved organ(s)

Table 1. Hernia Grading System as described by the Ventral Hernia Working Group ${ }^{[8]}$

\begin{tabular}{lll}
\hline Hernia Grading System & & \\
\hline Grade 1 (low risk) & \multicolumn{1}{c}{ Grade 2 (comorbid) } & Grade 3 (contaminated) \\
\hline No history of wound complications & . Comorbid conditions (DM, smoking, & . Presence of nearby stoma \\
Noevidence of contamination & immunocompromised, COPD, obesity) & . History of wound infection \\
No comorbidities & - No evidence of contamination & -Violation of Gl tract \\
& & -Septic dehiscence \\
& & -Infected mesh \\
& -Active infection \\
\hline
\end{tabular}

In addition to the grading system developed by VHWG, multiple other tools are used for risk stratification of complications developed after ventral hernia repair. Such models include the American College of Surgeons National Surgical Quality Improvement Program (ACS-NSQIP), European Hernia Society (EHS), The HERNIAscore, and Ventral Hernia Risk Score (VHRS). The HERNIAscore developed by Goodenough et al ${ }^{[9]}$ helps to identify risk factors for ventral incisional hernia formation. Laparotomy, hand-assisted laparoscopy, COPD, and BMI greater than 25 are all independent risk factors for hernia 
development. Depending on the score from zero to six, the risk for hernia ranges from $5.5 \%$ to $55 \%{ }^{[9]}$. The HERNIAscore has also been improved to include length of incision, COPD, and prior abdominal operations $^{[10]}$.

VHRS is an additional tool available for risk assessment of surgical site infection (SSI). Based on VHRS, risk factors for SSI include concomitant hernia repair, raising of skin flaps, wound Class 4 (i.e., dirty), BMI greater than 40 , and ASA Class 3 or greater ${ }^{[11]}$. Both the HERNIAscore and VHRS have been externally validated and shown to be more effective in identifying SSI/SSO risk when compared to other risk models. Overall, while some models are helpful, not all have been internally and externally validated. Validated grading scales can help counsel patients and set realistic expectations for possible complications following ventral hernia repair.

\section{CURRENT TECHNIQUES IN HERNIA MANAGEMENT}

\section{Abdominal wall closure in transplant patients}

Surgical closure of an abdomen following solid organ transplant is a critical aspect of the transplant, as a failure of closure greatly increases the risk for graft failure and infection. Primary closure of the abdominal wall is, of course, ideal, however cannot be achieved in approximately $20 \%$ of patients ${ }^{[12]}$. A common reason for failure of primary closure is lack of intra-abdominal domain. Some initial loss of domain is transient from edema and resolves with diuresis, etc. A rush to primary closure only increases the risk of dehiscence and wound breakdown. Patients may also present with presence of fistulae, ostomies, extensive scar tissue, significant skin lesions from healed fistulae/wounds, or even a frozen abdomen secondary to dense adhesions from multiple prior procedures, thus making primary closure challenging or even impossible to perform. In these patients with such anatomic complexities, an open approach may be favorable.

Significant tension on the abdominal wall from closure in patients with loss of domain can also lead to abdominal compartment syndrome, which in a newly transplanted graft can lead to graft failure. Foley catheters are commonly left in place postoperatively, often to monitor urine output, but also to monitor bladder pressures in the setting of possible abdominal compartment syndrome. It is imperative that the patient is intubated, paralyzed, and fully supine in order to obtain accurate bladder pressures. Increased abdominal pressure that does not cause compartment syndrome can still impede flow within the inferior vena cava and decrease portal venous flow to the liver. This is a known risk factor for graft failure following liver transplantation. Low flow states can also lead to poor venous return, resulting in decreased cardiac output and perfusion. Increased abdominal pressure after fascial closure can also lead to respiratory compromise, especially in patients with loss of domain. Thus, it is important to communicate with the anesthesia team intraoperatively to monitor peak pressures after closure, as some patients may require continued intubation in the immediate postoperative period. Elevated ventilatory pressure or changes in peak pressures may also serve as a real time indication of impending compartment syndrome so our group routinely solicits feedback from anesthesia throughout closure.

\section{Panniculectomy and transplantation}

Obesity remains a significant risk factor for morbidity after abdominal surgery. Transplantation in general carries a higher degree of morbidity among obese patients. Large overhanging skin folds create a moist environment that increases the risk of postoperative infection. Furthermore, the large amount of subcutaneous tissue that must be traversed and potentially undermined in patients undergoing transplant has a higher risk of necrosis and infection. A large pannus may also create additional tension and stress on the incision, which may lead to separation of wound edges ${ }^{[13]}$. Individual centers have specific protocols and regulations regarding optimal BMI prior to transplantation. While there is no consensus, some centers suggest having a BMI less than 30 to decrease the risk of perioperative complications ${ }^{[14]}$. A prophylactic panniculectomy can be performed prior to transplant to enable obese patients to meet criteria 
for transplantation. Performing panniculectomy in advance will allow patients to be better optimized for transplantation and allow them to heal without the influence of immunosuppression. However, this staged approach may add cost relative to simultaneous panniculectomy and transplant. It should be acknowledged that, while the pre-transplant panniculectomy patients are often not pharmacologically immunosuppressed, they are still relatively immunosuppressed due to their end-stage organ dysfunction.

Ngaage et al ${ }^{[15]}$ discussed panniculectomy at the time of living donor renal transplantation. In their study, panniculectomy was performed prior to standard oblique muscle cutting incisions such as a Gibson or Rutherford-Morison incision for transplantation. Among 58 patients with an average BMI of 35.2 who underwent simultaneous panniculectomy and renal transplant, the authors noted comparable rates of wound complications when compared to panniculectomy without transplant. Furthermore, there was no significant difference in allograft complications compared to the standard two-staged procedure, nor was there a significant difference in length of hospital stay ${ }^{[15]}$.

In the single-stage operation by Ngaage et al. ${ }^{[15]}$, the reconstructive surgeon mobilizes, undermines, and reflects the redundant soft tissue, exposing entirely the underlying fascia. This exposure provides access to the abdominal fascia and then facilitates closure of the defect. Simultaneous panniculectomy appears to be performed without a significant increase in wound healing morbidity even though these single-stage patients must face the challenge of healing after induction and maintenance of immunosuppression ${ }^{[15]}$. It is noteworthy that the study by Ngaage et al. ${ }^{[15]}$ did not have homogeneous immunosuppressive regimens, which may have skewed the interpretation of wound complication rates.

\section{Use of mesh in post-transplant incisional hernia repair}

The optimal type of mesh to use in hernia repair - and hernia prevention - is widely debated. However, there has recently been a greater shift towards the use of biologic mesh over synthetic mesh. Biological materials such as human acellular dermal matrix (HADM) and porcine acellular dermal matrix (PADM) have multiple advantages over their synthetic counterparts. Biologic mesh provides a scaffold that allows cellular and vascular ingrowth, thus benefitting from possible incorporation into native abdominal wall tissue $e^{[9]}$. The vascularization of acellular dermal matrix (ADM) assists the immune system in combating bacterial contamination. Furthermore, ADM has low antigenic potential and elicits a milder host inflammatory response compared to synthetic mesh. This allows for a less adhesiogenic environment in comparison to prosthetic mesh, making ADM desirable for intraperitoneal use ${ }^{[16]}$. ADM also contains collagen cross-linkages, which provide strength and resistance to mechanical stress ${ }^{[16]}$. Among popular acellular dermal matrices (AlloDerm, FlexHD, Strattice, Neoform, and DermACELL), AlloDerm (a HADM) has been shown to be more effective against bacterial growth. This was supported by Fahrenbach et al. ${ }^{[17]}$ who showed that AlloDerm was resistant to penetration with S. aureus and S. pyogenes. However, AlloDerm contains more elastin than its porcine equivalent; thus, it is more prone to stretching and can lead to recurrence, bulging and weakness when used in the abdominal wall. ${ }^{17}$ Generally speaking, non-crosslinked porcine $\mathrm{ADM}$ has been found to be beneficial in higher risk patients, including the transplant population.

Multiple studies have shown that mesh implantation during incisional hernia repair can decrease the risk of hernia recurrence without increasing risk for infection in the transplant population ${ }^{[2,5]}$. A study published by our group compared clinical outcomes of incisional hernia repair in 87 patients receiving solid organ transplants in which PADM, HADM, or synthetic mesh was utilized. We found that patients whose hernias were repaired using synthetic mesh had a significantly higher incidence of wound infection, hernia recurrence, and mesh removal ${ }^{[1]}$. Another study observing 104 patients undergoing renal, liver, or pancreas transplants had a statistically significant odds ratio of 11 and 8.7, respectively, for wound infection and mesh removal when comparing HADM with synthetic mesh ${ }^{[18]}$. It is important to note that neither of these studies was blinded, therefore inherent selection bias is possible in both. 
As mentioned, HADM has a greater tendency to stretch over time, given the higher relative content of elastin within the matrix when compared to Porcine versions. Some studies have documented increased rates of bulging and hernia recurrence in patients with large complex hernias repaired with HADM. Our group found that, unlike its human analog, PADM resists early stretching after implantation while still providing comparable tensile strength, thus demonstrating a clinically relevant but statistically nonsignificant benefit to using PADM in abdominal wall reconstruction in the transplant population ${ }^{[1]}$. Despite advantages and disadvantages to using either HADM or PADM, at this time, there is little evidence showing a significant benefit of one type of ADM over another.

\section{Operative approaches}

Multiple operative techniques are currently used by general, plastic, and transplant surgeons to repair incisional hernias in transplant patients. Primary suture repair is often limited to primary ventral hernias with small defects less than $3 \mathrm{~cm}$. However, some surgeons still opt for mesh reinforcement in small repairs due to higher rates of recurrence in this patient population. Repair with mesh is recommended for repair of incisional hernias larger than $2 \mathrm{~cm}$ in a non-infected field ${ }^{[19]}$. Laparoscopic intraperitoneal onlay mesh (IPOM) is a commonly-used repair technique for small- to medium-sized hernias, although it has been described as useful in facial defects up to $10 \mathrm{~cm}^{[19]}$. IPOM may also be useful in transplant patients who have undergone multiple abdominal surgeries and either lack a viable peritoneum or are missing a portion of posterior rectus sheath, thus making them ineligible for retro-rectus repair. In any mesh repair, it is important to ensure adequate overlap of the defect by at least $3-5 \mathrm{~cm}$ to decrease the risk of recurrence and to offload tension ${ }^{[19]}$. Furthermore, recognizing there is a growing adoption of posterior approaches, there still may be benefit for a modified onlay approach in properly selected patients, as described by the authors $^{[20]}$. In this component separation onlay approach, PADM is anchored to the donor site cut edge of external oblique on either side of the defect, thus providing a spanning and load-sharing structure that reinforces the midline closure with low rate of hernia recurrence and surgical site occurrence ${ }^{[20]}$.

Open sublay (e.g., retro-rectus) mesh repair has been shown in some studies to have similar recurrence rates as compared to IPOM, but these sublayed repairs are notable for increased perioperative morbidity and hospital length of stay ${ }^{[21]}$. However, the retro-rectus approach has several advantages when compared to IPOM and is being used more frequently in incisional hernia repairs in both post-transplant and general populations. Retro-rectus hernia repair, such as the Rives-Stoppa technique, maintains extraperitoneal access, which allows the surgeon to avoid contact with intraperitoneal adhesions and other organs, including the graft. By existing outside of the peritoneum, this technique also prevents the formation of adhesions that may hinder future surgical interventions, including graft repair and re-transplantation.

Sublay repair is often used in conjunction with component separation techniques in order to restore the linea alba and medialize the rectus muscles. Component separation in post-transplant patients is often challenging, as the native planes are often distorted and scarred down. Additionally, as mentioned above, incisions such as Mercedes incisions have both horizontal and vertical components, which can further complicate plane dissection and hernia repair. Black et al. ${ }^{[22]}$ described a modified component separation for abdominal wall reconstruction in 19 liver and kidney transplant patients, where open perforator-sparing component separation techniques (e.g., posterior external oblique dissection) were used in conjunction with biologic mesh underlay. Their data show comparable rates of healing and long-term hernia recurrence compared to other techniques ${ }^{[22]}$. More research is needed to examine outcomes and hernia recurrence after sublay/retro-rectus repair in transplant patients.

While component separation is often a necessary maneuver to achieve fascial re-approximation, it is not without potential complications. Anterior component separation (ACS) requires creation of large subcutaneous flaps, which can disrupt the blood supply via transection of trans-rectus epigastric perforator 
to the overlying fat and skin, leading to skin necrosis and its downstream sequelae. As mentioned above, this dissection also creates large flaps, which often harbor enormous areas of dead space and associated fluid collections. It should be noted that perforator-preserving ACS techniques have been described with improved outcomes. A posterior component separation (PCS) allows for less subcutaneous dissection and also may be preferred in patients who have undergone prior ACS repairs. In cases where patients lack adequate retro-rectus space, a unilateral or bilateral transverse abdominis release (TAR) may be required to minimize tension while avoiding the neurovascular bundles at the lateral border of the rectus muscle. As a general rule, we recommend that the retro-rectus space be at least twice the size of the defect transversely in order to undergo repair without the need for TAR. An advantage of TAR is the ability to create a wide retro-muscular space that can extend to the psoas muscles, Cooper's ligaments, and the central tendon of the diaphragm.

A meta-analysis from 2018 comparing open ACS to PCS with TAR showed similar hernia recurrence rates and wound complication rates; however, both larger and comparative studies still need to be performed ${ }^{[23]}$. It is difficult to discern which approach is superior. Ultimately, the choice of repair is multifactorial and will vary based on patient comorbidities, hernia morphology, and surgeon experience.

Finally, robotic-assisted hernia repair can aid in abdominal wall reconstruction for complex and large-sized hernias. A robotic approach may facilitate dissection and component separation in the setting of dense adhesions and complicated post-transplant anatomy. It is our recommendation that such cases should be performed at hernia centers that specialize in both complex hernia repair as well as transplant surgery such that, if there are complications, the transplant team is readily available.

Significant variability exists between size criteria and the appropriateness of individual techniques among surgeons; therefore, surgeon experience, patient factors, and hernia morphology must be considered when choosing how to repair incisional hernia in solid organ transplant patients. Currently, there are no definitive algorithms for hernia repair in this population for multiple reasons. First, a reparative approach can be determined by size of fascial defect, patient risk factors, or even hernia stages. There is no consensus as to which category is preferred in terms of stratifying patients to follow a certain algorithmic path. It is this lack of a universal classification system that has prevented development of algorithms in the past and this remains true today. Secondly, there is a great deal of variation in hernia repair technique that differs among institutions and surgeons, based on individual operator preference, experience, and available resources. What may be an acceptable approach at one institution may be deemed outdated, less desirable, or even impossible at another. Finally, there is so much nuance regarding incisional hernias in transplant patients - hernia size and location, degree of immunosuppression, comorbidities, loss of domain, and history of previous surgeries and hernia repairs to name a few - that there are infinite possible approaches to repair. Algorithms fail to be useful when they are too convoluted. This sentiment is best stated by Malangoni and Rosen in the 20th Edition of the Sabiston Textbook of Surgery: "The absence of a universal classification system has hindered comparisons within the literature and at meetings, indirectly delaying meaningful conversations about repair techniques and prosthetic choice. The TNM model for cancer staging is an enviable model to strive for in hernia repair" ${ }^{\text {"24] }}$. As of now, a comparable model does not exist.

\section{Autologous tissue flaps}

While not common, some transplant patients' hernias are so complicated, or have failed one or more biological mesh repairs, that surgeons must turn to autologous tissue flaps such as tensor fascia lata or thigh flaps. These autologous flaps were performed more frequently in the past and are now mostly of historical interest because complicated hernias are so effectively managed by biological mesh and novel methods of tissue expansion that complicated flap procedures are rarely necessary. 


\section{Abdominal wall vascularized composite allograft}

Multiple techniques have been described to help achieve abdominal wall closure including component separation, Gortex patch, use of biologic mesh (as described above), autologous flaps, and more recently abdominal wall transplantation. Abdominal wall transplantation, more commonly known as abdominal wall vascularized composite allograft (AW-VCA), is a modern alternative to abdominal wall closure which is typically reserved for truly complex defects. Utilization of AW-VCA can be separated into three categories: (1) patients receiving AW-VCA in conjunction with intestinal transplant; (2) patients receiving AW-CVA who already have a visceral organ transplant such as liver, kidney, or pancreas; and (3) AW-CVA performed as an isolated soft tissue transplant, which has shown promise in cadaver models. It is important to note that in non-transplant patients performing AW-VCA will subject patients to lifelong immunosuppression. On the contrary, for transplant patients, they are already immune suppressed.

AW-VCA can be either partial or full-thickness. In full-thickness transplants, the abdominal wall including the peritoneum, rectus abdominis muscle(s), and variable amounts of oblique muscle, as well as skin and soft tissue - are harvested en bloc. Partial-thickness reconstruction involves vascularized or nonvascularized fascia in patients with adequate skin cover, but insufficient or inadequate fascia ${ }^{[25]}$.

Two techniques have been described for abdominal wall transplants. In the conventional method, blood supply from the donor is taken from the inferior epigastric vessels, which are left in continuity with the femoral and iliac vessels and then anastomosed to the recipient's common iliac artery and vein ${ }^{[12]}$. The second method uses a microvascular technique to anastomose the donor's inferior epigastric vessels to those of the recipient ${ }^{[26]}$. The fascia of the donor is then sutured to the abdominal wall fascia of the recipient. A layered closure involving the subcutaneous tissues and skin of the donor and recipient is then performed, thereby completing graft integration. In both techniques, the abdominal donor graft is taken from a beating-heart donor. Procurement ideally is taken from the same donor of the abdominal organs. Interestingly, there have been reports of procurement from abdominal wall-specific donors.

Levi's group in 2003 was the first group to report their experience in a nine-case series of abdominal wall transplants ${ }^{[12]}$. This series included adults and children and was later pooled with additional patients in $2009^{[27]}$. Noted indications for intestinal transplant in their series included Gardner's syndrome, trauma, Churg-Strauss vasculitis, and intestinal motility disorders such as Hirschsprung and pseudo-obstruction ${ }^{[12]}$. Since the initial report in 2003, there have been 35 cases of AW-VCA transplantation with flap survival rates as high as $88 \%$ with immunosuppression ${ }^{[25,28]}$.

Postoperative immunosuppression protocols are center-specific. These regimens commonly include antibody induction with maintenance therapy using tacrolimus and/or mycophenolate mofetil. Steroids may also be used during maintenance therapy. One study noted a rejection rate of $17.7 \%$ among a cohort of 17 patients ${ }^{[29]}$. Rejection of the graft is often accompanied by a maculopapular rash or skin breakdown. This is in part due to the strong antigen surveillance role played by Langerhan cells within the skin, which may demonstrate signs of rejection when other composite tissues may not. Early skin changes on the abdominal wall are an indication of rejection and thus treatments such as steroid boluses and medication adjustments can be initiated sooner. Rejection may respond to topical immunosuppressive application as well or in conjunction with oral steroids The added benefit of a skin rash is being a sentinel marker of infection that provides a visual which patients may share with their transplant team, prompting intervention sooner than would be otherwise. Furthermore, because transplanted skin can act as a marker of visceral rejection via a rash, patients may have immunosuppressive regimens titrated down as long as such symptoms do not $\operatorname{occur}^{[30]}$. It is worth noting that multiple studies have reported that deaths after transplantation have not been directly related to abdominal wall transplants. 
Since the results published by Levi et al. ${ }^{[12]}$, numerous research studies have focused on AW-CVA as an isolated soft tissue transplant without viscera. Quigley et al. ${ }^{[31]}$ (2013) conducted complete isolated AW-VCA transplantation with femoral micro-anastomosis in rats. They used an immunosuppressive regimen consisting of Tacrolimus and showed $100 \%$ graft survival at 100 days, however with limited chimerism ${ }^{[31]}$. Chimerism involves a patient having hematopoietic stem cells of both donor and recipient origin. A lower degree of chimerism in patients can lead to graft failure; however, it can be halted with immunosuppression. Over time, tolerance between the two cell lines can occur, known as mixed chimerism, leading to patients requiring less immunosuppression, making rejection less likely.

To date, there is no literature showing AW-VCA being performed outside of transplant patients as an isolated procedure. The application of isolated AW-VCA (without viscera) has significant potential in patients with large abdominal wall defects including multiple prior surgeries or trauma patients with profound loss of domain. These patients are often plagued by poor functional status and have undergone multiple attempts at repair. There is, however, some thought that the risks of lifelong immunosuppression outweigh the benefits of AW-VCA transplantation, which is in part why AW-VCA as an isolated soft tissue transplant has not yet been performed.

While AW-VCA is a solution for patients with profound domain loss, the transplanted abdominal walls are essentially defunctionalized mechanical retainers of abdominal contents. These denervated transplants lack all motor function which leads quickly to atrophy, fibrosis, and loss of strength. Thus, while the patient may have a vascularized abdominal wall, he or she may have significant physical dysfunction and deformity.

In cases where innervated AW-VCA have been attempted, innervation is often unsuccessful or incomplete. From promising results in rat models, there have been multiple cadaver studies describing innervated AW-VCA to preserve both motor and sensory functions. Using a component separation technique involving the external oblique, the thoracolumbar nerves can be isolated from the donor to allow for the AW-VCA to retain both sensation and motor function ${ }^{[32,33]}$. We designed and executed a cadaver study which combined the concepts of functional hernia repair with the goal of innervated abdominal wall allotransplantation through preparation of the graft using a "multi-layered" component separation technique that carefully identifies individual segmental intercostal nerves beneath the internal oblique muscle. By preserving the nerve supply, the rectus muscle can theoretically remain innervated after transplant, which would allow for faster functional rehabilitation, increased strength, and decreased complications from a denervated abdominal wall such as bulge or hernia. In this study, they harvested the lowermost portion of the ribcage with the innervated soft tissue abdominal wall specimens in two cadavers. This required plates and screws for osteosynthesis but came with the advantage of presence of bone and bone marrow in the graft, which is thought to potentially promote immunogenic chimerism and thus decrease immunosuppressive requirements ${ }^{[32]}$.

\section{CONCLUSION}

Management of incisional hernia remains very complex, even more so in the post-transplant population. When planning any hernia repair in this patient population, one must consider patient comorbidities and risk factors, hernia morphology, and surgeon experience. Furthermore, a multidisciplinary approach should be used regarding each patient's immunosuppression regimen, ideally including a transplant pharmacologist. While a variety of repair options exist, it has not been possible to create an algorithmic approach to such a heterogeneous population. Therefore, each patient must be approached systematically to determine the most appropriate repair. Lastly, AW-VCA is an option for very complex defects or in patients with significant loss of domain, and new techniques may allow innervation in the transplanted abdominal wall. 


\section{DECLARATIONS}

\section{Author's contributions}

Made substantial contributions to the literature review, writing, and editing of this manuscript: Singh D, Holton L, Antognoli L, Choudhry S

Performed image creation and formatting: Antognoli L

Performed table creation and formatting: Choudhry S

\section{Availability of data and materials}

Not Applicable.

\section{Financial support and sponsorship}

None.

\section{Conflicts of interest}

Singh D is the consultant to $3 \mathrm{M}-\mathrm{KCI}$, Allergan, and Gore; Holton L is the consultant to $3 \mathrm{M}-\mathrm{KCI}$, Allergan, and Stryker; Antognoli L and Choudhry S have no conflicts of interest.

\section{Ethical approval and consent to participate}

Not Applicable.

\section{Consent for publication}

Not Applicable.

\section{Copyright}

(c) The Author(s) 2020.

\section{REFERENCES}

1. Gowda AU, Mcnichols CH, Asokan I, Matthews JA, Buckingham EB, et al. Porcine acellular dermal matrix for hernia repair in transplant patients. Ann Plast Surg 2016;77:674-7.

2. Garmpis N, Spartalis E, Schizas D, Patsouras D, Damaskos C, et al. Incisional hernias post liver transplantation: current evidence of epidemiology, risk factors and laparoscopic versus open repair. A review of the literature. In Vivo 2019;33:1059-66.

3. Ayvazoglu Soy EH, Kirnap M, Yildirim S, Moray G, Haberal M. Incisional hernia after liver transplant. Exp Clin Transplant 2017;15:185-9.

4. Fikatas P, Schoening W, Lee JE, Chopra SS, Seehofer D, et al. Incidence, risk factors and management of incisional hernia in a high volume liver transplant center. Ann Transplant 2013;18:223-30.

5. Hegab B, Abdelfattah MR, Azzam A, Al Sebayel M. The usefulness of laparoscopic hernia repair in the management of incisional hernia following liver transplantation. J Minim Access Surg 2016;12:58-62.

6. Piazzese E, Montalti R, Beltempo P, Bertelli R, Puviani L, et al. Incidence, predisposing factors, and results of surgical treatment of incisional hernia after orthotopic liver transplantation. Transplant Proc 2004;36:3097-8.

7. Aydin U, Pinar Y, Murat K. Optimal technique for abdominal fascial closure in liver transplant patients. Asian J Surg 2010;33:1-7.

8. Kanters AE, Krpata DM, Blatnik JA, Novitsky YM, Rosen MJ. Modified hernia grading scale to stratify surgical site occurrence after open ventral hernia repairs. J Am Coll Surg 2012;215:787-93.

9. Goodenough CJ, Ko TC, Kao LS, Nguyen MT, Holihan JL, et al. Development and validation of a risk stratification score for ventral incisional hernia after abdominal surgery: hernia expectation rates in intra-abdominal surgery (the HERNIA Project). J Am Coll Surg 2015;220:405-13.

10. Cherla DV, Moses ML, Mueck KM, Hannon C, Ko TC, et al. External validation of the HERNIAscore: an observational study. J Am Coll Surg 2017;225:428-34.

11. Liang MK, Goodenough CJ, Martindale RG, Roth JS, Kao LS. External validation of the ventral hernia risk score for prediction of surgical site infections. Surg Infect (Larchmt) 2015;16:36-40.

12. Levi DM, Tzakis AG, Kato T, Madariaga J, Mittal NK, et al. Transplantation of the abdominal wall. Lancet 2003;361:2173-6.

13. Troppmann C, Santhanakrishnan C, Kuo JH, Bailey CM, Perez RV, et al. Impact of panniculectomy on transplant candidacy of obese patients with chronic kidney disease declined for kidney transplantation because of a high-risk abdominal panniculus: a pilot study. Surgery 2016;159:1612-22. 
14. Dudley C, Harden P. Assessment of the potential kidney transplant recipient. Available from: https://renal.org/wp-content/ uploads/2017/06/assessment-of-the-potential-kidney-transplant-recipient-5th-edition-1.pdf. [Last accessed on 9 Sep 2019]

15. Ngaage LM, Elegbede A, Tadisina KK, Gebran SG, Masters BM, et al. Panniculectomy at the time of living donor renal transplantation: an 8-year experience. Am J Transplant 2019;19:2284-93.

16. Coccolini F, Catena F, Bertuzzo VR, Ercolani G, Pinna A, et al. Abdominal wall defect repair with biological prosthesis in transplanted patients: single center retrospective analysis and review of the literature. Updates Surg 2013;65:191-6.

17. Fahrenbach EN, Qi C, Ibrahim O, Kim JY, Alam M. Resistance of acellular dermal matrix materials to microbial penetration. JAMA Dermatol 2013;149:571-5.

18. Brewer MB, Rada EM, Milburn ML, Goldberg NH, Singh DP, et al. Human acellular dermal matrix for ventral hernia repair reduces morbidity in transplant patients. Hernia 2011;15:141-5.

19. Earle D, Roth JS, Saber A, Haggerty S, Bradley JF 3rd, et al; SAGES Guidelines Committee. SAGES guidelines for laparoscopic ventral hernia repair. Surg Endosc 2016;30:3163-83.

20. Singh DP, Zahiri HR, Gastman B, Holton LH 3rd, Stromberg JA, et al. A modified approach to component separation using biologic graft as a load-sharing onlay reinforcement for the repair of complex ventral hernia. Surg Innov 2014;21:137-46.

21. Alizai PH, Lelaona E, Andert A, Neumann UP, Klink CD, et al. Incisional hernia repair of medium- and large-sized defects: laparoscopic IPOM versus open SUBLAY technique. Acta Chir Belg 2019;119:231-5.

22 Black CK, Zolper EG, Walters ET, Wang J, Martinez J, et al. Utility of a modified components separation for abdominal wall reconstruction in the liver and kidney transplant population. Arch Plast Surg 2019;46:462-9.

23 Hodgkinson JD, Leo CA, Maeda Y, Bassett P, Oke SM, et al. A meta-analysis comparing open anterior component separation with posterior component separation and transversus abdominis release in the repair of midline ventral hernias. Hernia 2018;22:617-26.

24. Malangoni MA, Rosen MJ, Hernias. In: Townsend Jr CM, Beauchamp RD, Evers BM, Mattox KL, editors. Sabiston Textbook of Surgery: The Biological Basis of Modern Surgical Practice. 20th ed. Philadelphia, PA: Saunders Elsevier; 2017.pp.1109-10.

25. Giele H, Vaidya A, Reddy S, Vrakas G, Friend P. Current state of abdominal wall transplantation. Curr Opin Organ Transplant 2016;21:159-64.

26. Cipriani R, Contedini F, Santoli M, Gelati C, Sgarzani R, et al. Abdominal wall transplantation with microsurgical technique. Am J Transplant 2007;7:1304-7.

27. Selvaggi G, Levi DM, Cipriani R, Sgarzani R, Pinna AD, et al. Abdominal wall transplantation: surgical and immunologic aspects. Transplant Proc 2009;41:521-2.

28. Park SH, Eun SC. Abdominal wall transplant surgery. Exp Clin Transplant 2018;16:745-50.

29. Berli JU, Broyles JM, Lough D, Shridharani SM, Rochlin D, et al. Current concepts and systematic review of vascularized composite allotransplantation of the abdominal wall. Clin Transplant 2013;27:781-9.

30. Barnes J, Issa F, Vrakas G, Friend P, Giele H. The abdominal wall transplant as a sentinel skin graft. Curr Opin Organ Transplant 2016;21:536-40.

31. Quigley MA, Fletcher DR, Zhang W, Nguyen VT. Development of a reliable model of total abdominal wall transplantation. Plast Reconstr Surg 2013;132:988-94.

32. Broyles JM, Berli J, Tuffaha SH, Sarhane KA, Cooney DS, et al. Functional abdominal wall reconstruction using an innervated abdominal wall vascularized composite tissue allograft: a cadaveric study and review of the literature. J Reconstr Microsurg 2015;31:39-44.

33. Hunter DJ, Hankinson SE, Laden F, Colditz GA, Manson JE, et al. Plasma organochlorine levels and the risk of breast cancer. N Engl J Med 1997;337:1253-8. 